\title{
Evolutionary Computing Optimization for Parameter Determination and Feature Selection of Support Vector Machines
}

\author{
Sheng Ding \\ School of Remote Sensing and Information Engineering \\ Wuhan University \\ Wuhan, 430079, China \\ College of Computer Science and Technology \\ Wuhan University of Science and Technology \\ Wuhan, 430065, China \\ dingwhu@gmail.com
}

\author{
Xiaoming Liu \\ College of Computer Science and Technology \\ Wuhan University of Science and Technology \\ Wuhan, 430065, China \\ 1xmspace@gmail.com
}

\begin{abstract}
Support vector machine (SVM) is a popular pattern classification method with many diverse applications. Kernel parameter setting in the SVM training procedure, along with the feature selection, significantly influences the classification accuracy. This study simultaneously determines the parameter values while discovering a subset of features, increasing SVM classification accuracy. The study focuses two evolutionary computing approaches to optimize the parameters of SVM: particle swarm optimization (PSO) and genetic algorithm (GA). And we combine the two evolutionary methods with SVM to choose appropriate subset features and SVM parameters, experimental results demonstrate that the classification accuracy surpass traditional grid searching approach. Also the paper compares PSO with GA method based SVM classification and they have similar results.
\end{abstract}

Keywords-support vector machine (SVM); genetic algorithm (GA); particle swarm optimization (PSO) ;optimization.

\section{INTRODUCTION}

Support vector machines (SVM) were first suggested by Vapnik [1] and have recently been used in a range of problems including pattern recognition, bioinformatics and text categoryzation. SVM classifies data with different class labels by determining a set of support vectors that are members of the set of training inputs that outline a hyperplane in the feature space. When using SVM, two problems are confronted: how to choose the optimal input feature subset for SVM, and how to set the best kernel parameters. These two problems are crucial, because the feature subset choice influences the appropriate kernel parameters and vice versa [2].Therefore, obtaining the optimal feature subset and SVM parameters must occur simultaneously.

Feature selection is used to identify a powerfully predictive subset of fields within a database and reduce the number of fields presented to the mining process. By extracting as much information as possible from a given data set while using the smallest number of features, we can save significant computation time and build models that generalize better for unseen data points. In addition to the feature selection, proper parameters setting can improve the SVM classification accuracy. The parameters that should be optimized include penalty parameter $C$ and the kernel function parameters such as the gamma $(\gamma)$ for the radial basis function (RBF) kernel. To design a SVM, one must choose a kernel function, set the kernel parameters and determine a soft margin constant $C$ (penalty parameter). The Grid algorithm is an alternative to finding the best $C$ and gamma $(\gamma)$ when using the RBF kernel function. However, this method is time consuming and does not perform well [3]. Moreover, the Grid algorithm cannot perform the feature selection task.

This study attempts to increase the classification accuracy rate by employing two evolutionary computing optimizationbased approaches: genetic algorithm (GA) and particle swarm optimization (PSO) in SVM. This novel approaches are termed PSO-SVM and GA-SVM. The developed approaches not only tune the parameter values of SVM, but also identify a subset of features for specific problems, maximizing the classification accuracy rate of SVM. This makes the optimal separating hyperplane obtainable in both linear and non-linear classification problems

The remainder of this paper is organized as follows. Section II reviews pertinent literature on SVM and the feature selection. Section III then describes basic GA concept and GASVM model of feature selection and parameter optimization. Next, Section IV then describes in detail the developed PSOSVM approach for determining the parameter values for SVM with feature selection. Section $\mathrm{V}$ compares the experimental results with those of existing approaches. Conclusions are finally drawn in Section VI, along with recommendations for future research.

\section{LITERATURE REVIEW}

Several kernel functions help the SVM in obtaining the optimal solution. The most frequently used such kernel functions are the polynomial, sigmoid and radial basis kernel function (RBF). The RBF is generally applied most frequently, because it can classify multi-dimensional data, unlike a linear kernel function. Additionally, the RBF has fewer parameters to set than a polynomial kernel. RBF and other kernel functions have similar overall performance. Consequently, RBF is an 
effective option for kernel function. Therefore, this study applies an RBF kernel function in the SVM to obtain optimal solution. Two major RBF parameters applied in SVM, $C$ and $\gamma$, must be set appropriately. Parameter $C$ represents the cost of the penalty. The choice of value for $C$ influences on the classification outcome. If $C$ is too large, then the classification accuracy rate is very high in the training phase, but very low in the testing phase. If $C$ is too small, then the classification accuracy rate is unsatisfactory, making the model useless. Parameter $\gamma$ has a much greater influence on classification outcomes than $C$, because its value affects the partitioning outcome in the feature space. An excessively large value for parameter $\gamma$ results in over-fitting, while a disproportionately small value leads to under-fitting. Grid search [4] is the most common method to determine appropriate values for $C$ and $\gamma$. Values for parameters $C$ and $\gamma$ that lead to the highest classification accuracy rate in this interval can be found by setting appropriate values for the upper and lower bounds (the search interval) and the jumping interval in the search. Nevertheless, this approach is a local search method, and vulnerable to local optima. Additionally, setting the search interval is a problem. Too large a search interval wastes computational resource, while too small a search interval might render a satisfactory outcome impossible.

In addition to the commonly used grid search approach, other techniques are employed in SVM to improve the possibility of a correct choice of parameter values. Pai and Hong [5] proposed an SA-based approach to obtain parameter values for SVM, and applied it in real data; however, this approach does not address feature selection, and therefore may exclude the optimal result. As well as the two parameters $C$ and $\gamma$, other factors, such as the quality of the feature's dataset, may influence the classification accuracy rate. For instance, the correlations between features influence the classification result. Accidental removal of important features might lower the classification accuracy rate. Additionally, some dataset features may have no influence at all, or may contain a high level of noise. Removing such features can improve the searching speed and accuracy rate.

It is worth underlining that the kernel-based implementation of SVMs involves the problem of the selection of multiple parameters, including the kernel parameters (e.g., the $\gamma$ and $p$ parameters for the Gaussian and polynomial kernels, respectively)and the regularization parameters $C$.

\section{THE DEVELOPED GA-SVM APPROACH}

\section{A. Genetic Algorithm}

The genetic algorithms are inspired by theory of evolution, it is type of an evolutionary computing. The problems are solved by an evolutionary process resulting in a fittest solution in genetic algorithm. A genetic algorithm (GA) is used to solve global optimization problems. The procedure starts from a set of randomly created or selected possible solutions, referred to as the population. Every individual in the population means a possible solution, referred to as a chromosome. Within every generation, a fitness function should be used to evaluate the quality of every chromosome to determine the probability of it surviving to the next generation; usually, the chromosomes with larger fitness have a higher survival probability. Thus, GA should select the chromosomes with larger fitness for reproduction by using operations like selection, crossover and mutation in order to form a new group of chromosomes which are more likely to reach the goal. This reproduction goes through one generation to another, until it converges on the individual generation with the most fitness for goal functions or the required number of generations was reached. The optimal solution is then determined.

GA coding strategies mainly include two sectors: one sector recommends the least digits for coding usage, such as binary codes, another one recommends using the real-valued coding based on calculation convenience and accuracy. Binary codes are adopted for the decision variables in solving the discrete problems, a suitable encoding scheme is needed to encode the chromosome of each individual, in our study, an encoding scheme is usually a binary string. We may define the length of bit string according the precision.

\section{B. GA-SVM Model}

As mentioned before, a kernel function is required in SVM for transforming the training data. This study adopts RBF as the kernel function to establish support vector classifiers, since the classification performance is significant when the knowledge concerning the data set is lacking. Therefore, there are two parameters, $C$ and $\gamma$, required within the SVM algorithm for accurate settings, since they are closely related to the learning and predicting performance. However, determining the values exactly is difficult for SVM. Generally, to find the best $C$ and $\gamma$, a given parameter is first fixed, and then within the value ranges another parameter is changed and cross comparison is made using the grid search algorithm. This method is conducted with a series of selections and comparisons, and it will face the problems of lower efficiency and inferior accuracy when conducting a wider search. However, GA for reproduction could provide the solution for this study. The scheme of an integration of GA and SVM is shown in Fig. 1, to establish a training and SVM classification model that can be used to determine optimized SVM parameters and subset features mask. Following the above scheme of the proposed GA-SVM model, Fig. 1 describes the operating procedure in this study.

A fitness function assesses the quality of a solution in the evaluation step. The crossover and mutation functions are the main operators that randomly impact the fitness value. Chromosomes are selected for reproduction by evaluating the fitness value. The fitter chromosomes have higher probability to be selected into the recombination pool using the roulette wheel or the tournament selection methods. New population replaces the old population using the elitism or diversity replacement strategy and forms a new population in the next generation. The evolutionary process operates many generations until termination condition is satisfied.

To implement our proposed approach, this research uses the RBF kernel function for the SVM classifier because the RBF kernel function can analysis higher-dimensional data and requires that only two parameters, $C$ and $\gamma$ be defined When the RBF kernel is selected, the parameters $(C$ and $\gamma)$ and features used as input attributes must be optimized using our proposed 
GA-based system. Therefore, the chromosome comprises three parts: $C, \gamma$ and the features mask. However, these chromosomes have different parameters when other types of kernel functions are selected. The binary coding system is used to represent the chromosome.

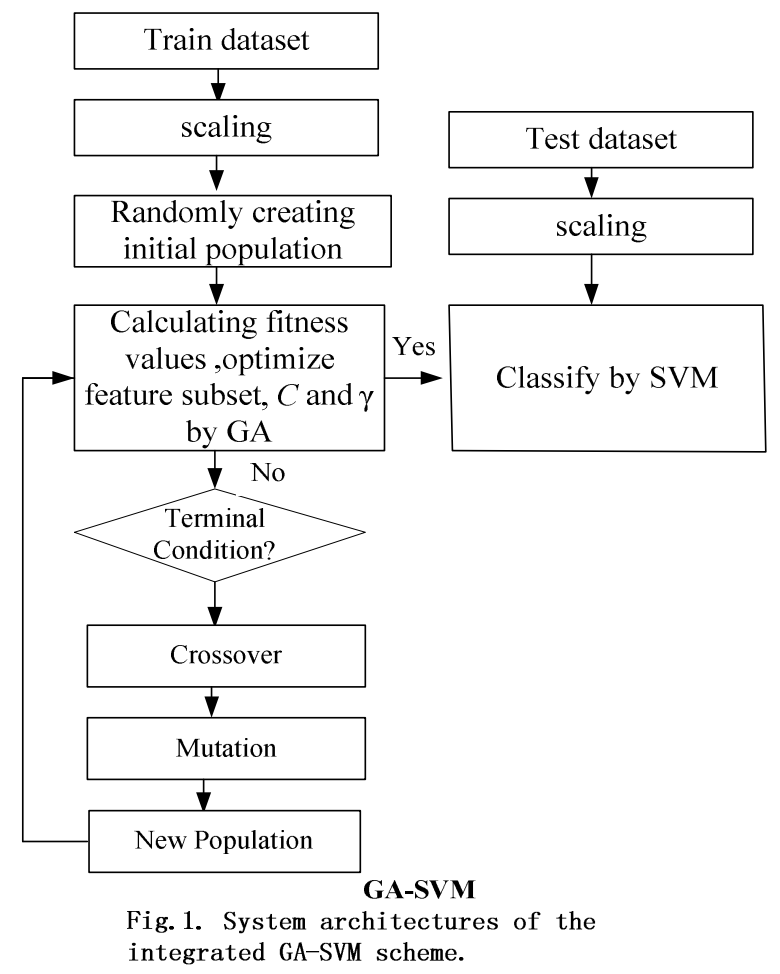

Fig.2 shows the binary chromosome representation of our design. In Fig. $2, g_{c}^{1} \sim g_{c}^{n_{c}}$ represents the value of parameter $C, g_{\gamma}^{1} \sim g_{\gamma}^{n_{\gamma}}$ represents the parameter value $\gamma$, and $g_{f}^{1} \sim$ $g_{f}^{n_{f}} \quad$ represents the feature mask. $n_{c}$ is the number of bits representing parameter $C, n_{\gamma}$ is the number of bits representing parameter $\mathrm{g}$, and $n_{f}$ is the number of bits representing the features. Note that we can choose $n_{c}$ and $n_{\gamma}$ according to the calculation precision required, and that $n_{f}$ equals the number of features varying from the different datasets. In Fig. 2, the bit strings representing the genotype of parameter $C$ and $\gamma$ should be transformed into phenotype. Note that the precision of representing parameter depends on the length of the bit string, and the minimum and maximum value of the parameter is determined by the user. For chromosome representing the feature mask, the bit with value ' 1 ' represents the feature is selected, and ' 0 ' indicates feature is not selected. In our study, classification accuracy, the numbers of selected features are the criteria used to design a fitness function. Thus, for the individual with high classification, a small number of features produce a high fitness value.

$$
g_{c}^{1} \ldots g_{c}^{i} \ldots g_{c}^{n_{c}} \quad g_{\gamma}^{1} \ldots g_{\gamma}^{i} \ldots g_{\gamma}^{n_{\gamma}} \quad g_{f}^{1} \ldots g_{f}^{i} \ldots g_{f}^{n_{f}}
$$

Fig.2. The chromosome comprise three parts: $C, \gamma$ and the features mask

\section{THE DEVELOPED PSO-SVM APPROACH}

Particle swarm optimization (PSO) [6] is an emerging population-based meta-heuristic that simulates social behavior such as birds flocking to a promising position to achieve precise objectives in a multi-dimensional space. Like evolutionary algorithms, PSO performs searches using a population (called swarm) of individuals (called particles) that are updated from iteration to iteration. To discover the optimal solution, each particle changes its searching direction according to two factors, its own best previous experience (pbest) and the best experience of all other members (gbest). They are called pbest the cognition part, and gbest the social part. Each particle represents a candidate position (i.e., solution). A particle is considered as a point in a $D$-dimension space, and its status is characterized according to its position and velocity. The $D$ dimensional position for the particle $i$ at iteration $\mathrm{t}$ can be represented as $x_{i}^{t}=\left\{x_{i 1}^{t}, x_{i 2}^{t}, \ldots x_{i D}^{t}\right\}$. Likewise, the velocity (i.e., distance change), which is also an D-dimension vector, for particle $i$ at iteration $t$ can be described as $v_{i}^{t}=\left\{v_{i 1}^{t}, v_{i 2}^{t}, \ldots v_{i D}^{t}\right\}$

Let $p_{i}^{t}=\left\{p_{i 1}^{t}, p_{i 2}^{t}, \ldots p_{i D}^{t}\right\}$ represent the best solution that particle $i$ has obtained until iteration $t$, and $p_{g}^{t}=\left\{p_{g 1}^{t}, p_{g 2}^{t}, \ldots p_{g D}^{t}\right\}$ denote the best solution obtained from $p_{i}^{t}$ in the population at iteration $t$. To search for the optimal solution, each particle changes its velocity according to the cognition and social parts as follows:

$$
\begin{aligned}
& V_{i d}^{t}=V_{i d}^{t-1}+c_{1} r_{1}\left(P_{i d}^{t}-x_{i d}^{t}\right)+c_{2} r_{2}\left(P_{g d}^{t}-x_{i d}^{t}\right) \\
& S\left(v_{i d}^{t+1}\right)=\frac{1}{1+e^{-v_{i d}^{t+1}}} \\
& \text { if }\left(\text { rand }<S\left(v_{i d}^{t+1}\right)\right) \text { then } X_{i d}^{t+1}=1 \text { else } X_{i d}^{t+1}=0
\end{aligned}
$$

$d=1,2, \cdots, D$ where $c_{1}$ indicates the cognition learning factor; $c_{2}$ indicates the social learning factor, and $r_{1}$ and $r_{2}$ are random numbers uniformly distributed in $U(0,1)$. Each particle then moves to a new potential solution based on the following equation: $X_{i d}^{t+1}=X_{i d}^{t}+V_{i d}^{t}, d=1,2, \cdots, D$ The basic process of the PSO algorithm is given as follows.

Step 1: (Initialization) Randomly generate initial particles.

Step 2: (Fitness) Measure the fitness of each particle in the population.

Step 3: (Update) Compute the velocity of each particle with Eq. (1).

Step 4: (Construction) For each particle, move to the 
next position according to Eq. (2).

Step 5: (Termination) Stop the algorithm if termination criterion is satisfied; return to Step 2 otherwise The iteration is terminated if the number of iteration reaches the predetermined maximum number of iteration.

Fig. 3 shows the flowchart for PSO-SVM

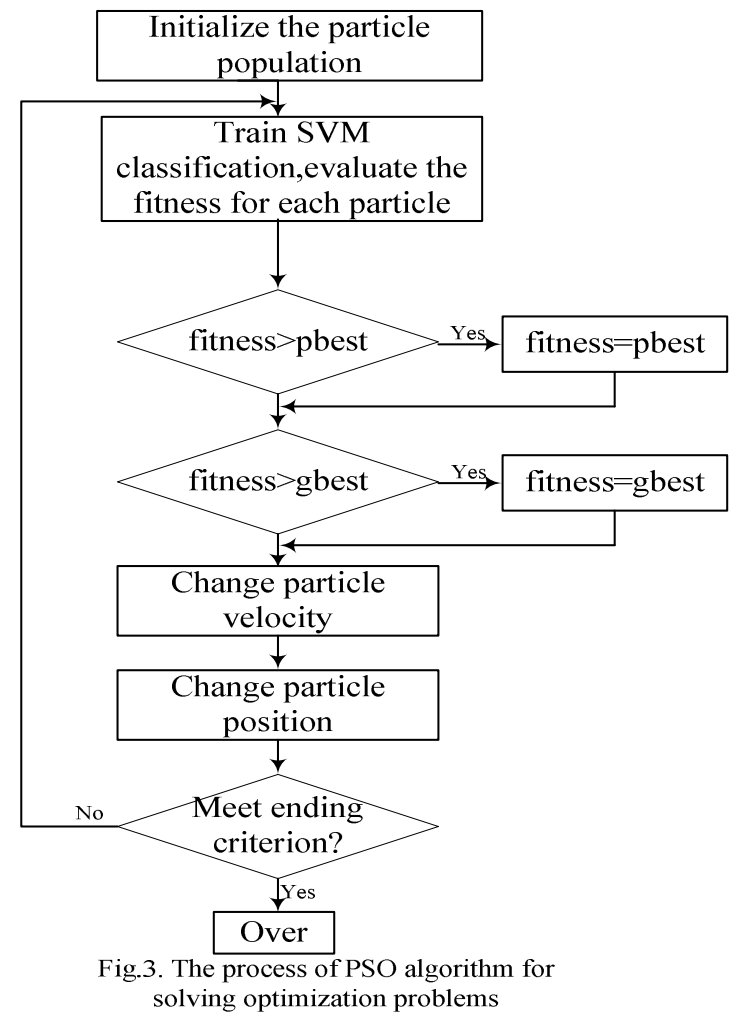

Based on the rules of particle swarm optimization, we set the required particle number first, and then the initial coding alphabetic string for each particle is randomly produced. In our study, we coded each particle to imitate a chromosome in a genetic algorithm.

\section{EXPERIMENT RESULTS}

To evaluate the classification accuracy of the proposed system in different classification tasks, we tried several realworld datasets from the UCI database [7]. These data sets have been frequently used as benchmarks to compare the performance of different classification methods the literature. These datasets consist of numeric and nominal attributes. To guarantee valid results for making predictions regarding new data, the dataset is further randomly partitioned into training sets and independent test sets via a $k$-fold cross validation. Each of the $k$ subsets acted as an independent holdout test set for the model trained with the remaining $k-1$ subsets. The advantages of cross validation are that all of the test sets were independent and the reliability of the results could be improved. The data set is divided into $k$ subsets for cross validation. A typical experiment uses $k=10$. Other values may be used according to the data set size. For a small data set, it may be better to set larger $k$, because this leaves more examples in the training set. This study used $k=10$, meaning that all of the data will be divided into 10 parts, each of which will take turns at being the testing data set. The other nine data parts serve as the training data set for adjusting the model prediction parameters.

Our implementation was carried out on the Matlab 7.0 development environment by extending the LIBSVM which is originally designed by Chang and Lin [8]. The empirical evaluation was performed on Intel Pentium Dual-Core CPU running at $1.6 \mathrm{GHz}$ and $2 \mathrm{G}$ RAM.

The Grid search algorithm is a common method for searching for the best $C$ and $\gamma$. Fig. 4 shows the process of Grid algorithm combined with SVM classifier. In the Grid algorithm, pairs of $(C, \gamma)$ are tried and the one with the best cross-validation accuracy is chosen. After identifying a 'better' region on the grid, a finer grid search on that region can be conducted. This research conducted the experiments using the proposed GA-SVM and PSO-SVM approaches and the Grid algorithm. The results from the proposed method were compared with that from the Grid algorithm. In all of the experiments 10 -fold cross validation is used to estimate the accuracy of each learned classifier.

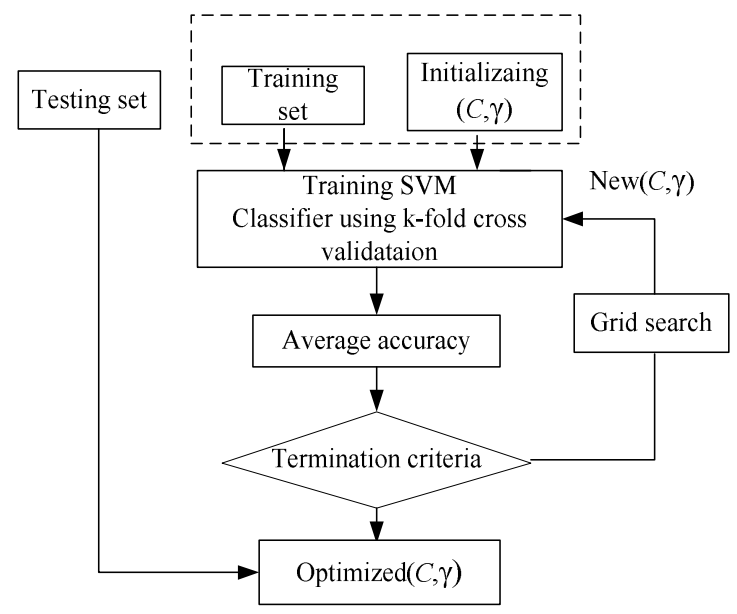

Fig .4. parameters setting using grid algorithm

The detail parameter setting for genetic algorithm is as the following: population size 20 , crossover rate 0.6 , mutation rate 0.05 , single-point crossover, roulette wheel selection, and elitism replacement, we set $n_{c}=12, n_{\gamma}=15$, the value of $\mathrm{n}_{f}$ depends on the experimental datasets stated in TABLE II. According to the fitness function and the number of selected features, we can compare different methods. The GA-SVM and PSO-SVM related parameters is described in TABLE III.

The termination criteria is that the generation number reaches generation 100 . The best chromosome is obtained when the termination criteria satisfy. Taking the German dataset, for example, over accuracy, number of selected features for each fold using GA-SVM approach, PSO-SVM approach and Grid algorithm are shown in TABLE I . For GA-SVM approach, its average accuracy is $87.08 \%$, and 
average number of features is 11.46 , and for PSO-SVM approach, the average accuracy is $85.47 \%$ and average number of features is 10.92 , but for Grid algorithm, its average accuracy is $81.46 \%$, and all 24 features are used.

To compare the two proposed evolutionary computing approaches with the Grid algorithm, as shown in TABLE II, Generally, compared with the Grid algorithm, the two proposed approaches have good accuracy performance with fewer features.

\begin{tabular}{|c|c|c|c|c|c|}
\hline \multirow[t]{2}{*}{ Fold \# } & \multicolumn{2}{|l|}{ GA-SVM } & \multirow{2}{*}{$\begin{array}{l}\text { PSO-SVM } \\
\text { overall } \\
\text { accuracy[\%] }\end{array}$} & \multicolumn{2}{|c|}{ Grid algorithm } \\
\hline & $\begin{array}{l}\text { overall } \\
\text { accuracy }\end{array}$ & $\begin{array}{l}\text { selected } \\
\text { features }\end{array}$ & & $\begin{array}{l}\begin{array}{c}\text { selected } \\
\text { features }\end{array} \\
\end{array}$ & $\begin{array}{c}\text { overall } \\
\text { accuracy[\%] }\end{array}$ \\
\hline 1 & 85 & 12 & 91 & 9 & 79 \\
\hline 2 & 86 & 8 & 90 & 12 & 80 \\
\hline 3 & 87 & 14 & 87 & 10 & 78 \\
\hline 4 & 88 & 11 & 86 & 11 & 81 \\
\hline 5 & 87 & 10 & 83 & 14 & 84 \\
\hline 6 & 84 & 9 & 84 & 13 & 80 \\
\hline 7 & 86 & 14 & 87 & 14 & 82 \\
\hline 8 & 88 & 14 & 86 & 10 & 82 \\
\hline 9 & 87 & 13 & 83 & 10 & 86 \\
\hline 10 & 89 & 10 & 88 & 7 & 82 \\
\hline 11 & 91 & 13 & 81 & 11 & 81 \\
\hline 12 & 85 & 13 & 82 & 11 & 84 \\
\hline 13 & 89 & 8 & 83 & 10 & 80 \\
\hline Avearage & 87.07692308 & 11.46153846 & 85.46153846 & 10.92307692 & 81.46153846 \\
\hline
\end{tabular}

TABLE II. EXPERIMENTAL RESULTS FOR TEST DATASET

\begin{tabular}{lcclcc}
\hline \multicolumn{1}{c}{ Names } & \multicolumn{2}{c}{ GA-SVM } & \multicolumn{2}{c}{ PSO-SVM } & Grid algorithm \\
\hline & $\begin{array}{l}\text { overall } \\
\text { accuracy }\end{array}$ & $\begin{array}{c}\text { selected } \\
\text { features }\end{array}$ & $\begin{array}{l}\text { overall } \\
\text { accuracy[\%] }\end{array}$ & $\begin{array}{l}\text { selected } \\
\text { features }\end{array}$ & $\begin{array}{c}\text { overall } \\
\text { accuracy[\%] }\end{array}$ \\
Australian & 88.2 & 5.87 & 91.34 & 6.23 & 87.14 \\
Heart disease & 92.05 & 7.53 & 95.12 & 5.76 & 85.47 \\
Vehicle & 88.43 & 9.34 & 93.02 & 11.5 & 83.33 \\
Sonar & 96.26 & 18.23 & 98.24 & 16.25 & 95.19 \\
breast cancer & 95.87 & 1.39 & 98.9 & 1.2 & 94.67 \\
\hline
\end{tabular}

TABLE III. PARAMETERS SETTING

\begin{tabular}{lclccc}
\hline PSO-SVM & & GA-SVM & \multicolumn{2}{l}{ Grid Algorithm } \\
\hline Parameter & value & Parameter & Value & Parameter & Value \\
Population size & 20 & Population size & 20 & C & $0 \ldots 2^{15}$ \\
Number of generations & 100 & Number of generations & 100 & $\gamma$ & $2^{-15} \ldots 1$ \\
Vmax & 4 & Probability of crossover & 0.6 & & \\
$C_{l,} C_{2}$ & 2 & Probability of mutation & 0.05 & & \\
\hline
\end{tabular}

\section{DISCUSSION AND CONCLUSTION}

This study presents two evolutionary computing optimization approaches, capable of searching for the optimal parameter values for SVM and a subset of beneficial features. This optimal subset of features and SVM parameters are then adopted in both training and testing to obtain the optimal outcomes in classification. Comparison of the obtained results with traditional Grid-based approach demonstrates that the developed PSO-SVM and GA-SVM approach have better classification accuracy with fewer features. After using feature selection in the experiment, the proposed approaches are applied to eliminate unnecessary or insignificant features, and effectively determine the parameter values, in turn improving the overall classification results, and the PSO-SVM approach is better than GA-SVM in most datasets.

Results of this study are obtained with an RBF kernel function. However, other kernel parameters can also be optimized using the same approach. Experimental results obtained from UCI datasets, other public datasets and realworld problems can be tested in the future to verify and extend this approach.

\section{ACKNOWLEDGMENT}

The author would like to thank Dr C.-J. Lin and C.-W. Hsu (National Taiwan University, Taipei, ROC) that the support vector machines were constructed with LIBSVM (Version 2.8) by them.

\section{REFERENCES}

[1] Vapnik Vladimir N. The Nature of Statistical Learning Theory[M] . Springer Verlag, New York, Inc, 2000.

[2] Fro"hlich, H., \& Chapelle, O. (2003). Feature selection for support vector machines by means of genetic algorithms. Proceedings of the 15th IEEE international conference on tools with artificial intelligence, Sacramento,CA, USA pp. 142-148.

[3] Hsu, C. W., \& Lin, C. J. (2002). A simple decomposition method for support vector machine. Machine Learning, 46(1-3), pp.219-314.

[4] Hsu, C.-W., Chang, C.-C., \& Lin, C.-J. (2003). A practical guide tosupport vector classification. Technical report, University of NationalTaiwan, Department of Computer Science and Information Engineering,July, pp. 1-12.

[5] Pai, P.-F., \& Hong, W.-C. (2005). Support vector machines with Simulated annealing algorithms in electricity load forecasting. Energy Conversionand Management, Vol . 46, pp.2669-2688.

[6] Kenned Y J, Eberhart R. Particle swarm optimization. In: IEEE International Conference on Neural Networks. Perth: IEEE Neural Networks Society, 1995: 1942-1948.

[7] Hettich, S., Blake, C. L., \& Merz, C. J. (1998). UCI repository of machine learning databases, Department of Information and Computer Science,University of California, Irvine, CA. http//www.ics.uci.edu/ $\sim$ mlearn/ MLRepository.html.

[8] Chih-Chung Chang and Chih-Jen Lin, LIBSVM: a library for support vector machines,2001. Software available at http://www.csie.ntu.edu.tw/ $\sim$ cjlin/libsvm 